\section{Unguentum lymphaticum bei Lympherkrankungen - heutiger Erkenntnisstand und Perspektiven}

\author{
M. Augustin, N. Denig, W. Vanscheidt,
} Universitäts-Hautklinik Freiburg

\section{Zusammenfassung}

Unguentum lymphaticum ist eine seit Jahrzehnten in der Lymphtherapie eingesetzte Salbe, die nach klinischer Erfahrung die physikalische Lymphbehandlung unterstützen kann. Sie enthält Zubereitungen aus sechs Pflanzen, deren Wirksamkeit auf Hauterkrankungen nur teilweise belegt ist. In-vitro-Studien deuten aber darauf hin, dass Anteile von Unguentum lymphaticum sowohl antiphlogistische wie auch antiödematöse und immunstimulierende Wirkungen haben. Es erscheint daher interessant, Unguentum lymphaticum zukünftig sowohl in kontrollierten klinischen Studien zu untersuchen wie auch die Einzelkomponenten an der Haut toxikologisch und pharmakologisch näher zu beschreiben.

\section{Hintergrund}

Unguentum lymphaticum ${ }^{\circledR}$ (Pharmasan, Freiburg) ist eine pflanzliche Salbe, die seit mehreren Jahrzehnten zur unterstützenden Behandlung von Lymphödemen eingesetzt wird [1]. Wenngleich diese Salbe bislang nicht in einer ausreichenden Zahl von kontrollierten klinischen Studien unter Evidenz-Gesichtspunkten überprüft worden ist, liegen dennoch zahlreiche positive Erfahrungsberichte vor. Nachfolgend sollen der derzeitige Kenntnisstand über Unguentum lymphaticum kurz zusammengefasst und mögliche weitergehende Untersuchungsansätze dargelegt werden.

\section{Zusammensetzung}

Unguentum lymphaticum besteht aus den alkoholischen Auszügen von sechs Pflanzen, eingearbeitet in eine Salbengrundlage: Conium maculatum (Schierling), Colchicum autumnale (Herbstzeitlose), Podophyllum peltatum (Maiapfel), Digitalis purpurea (Fingerhut), Hyoscyamus niger (Bilsenkraut) und Calendula officinalis (Ringelblume).

\section{Kurzportrait der pflanzlichen Inhaltsstoffe}

Conium maculatum (Gefleckter Schierling)

Diese in Mitteleuropa beheimatete Pflanze aus der Familie der Apiaceae ist wegen ihrer hohen Toxizität bei systemischer Wirkung - besonders durch die Vergiftung des Sokrates - weithin bekannt. Der Hauptinhaltsstoff, das Coniin, sowie weitere Piperidin-Alkaloide bewirken eine erhöhte Erregbarkeit der peripheren Nerven sowie in der Folge eine aufsteigende meduläre Lähmung des Atemzentrums [2].

Auf die peripheren Nervenfasern in der Haut scheint Coniin eine Nicotin-ähnliche Wirkung zu entfalten, möglicherweise im Sinne einer Vasokonstriktion. Da das Coniin auch durch Haut und Schleimhäute gut resorbiert wird, darf eine topische Applikation nur in niedrige Dosierungen erfolgen.

Colchicum autumnale (Herbstzeitlose)

Die Herbstzeitlose aus der Familie der Colchicaceae ist in Europa weit verbreitet. Neben dem Hauptalkaloid, Colchicin, sind viele weitere Alkaloide als Inhaltsstoffe bekannt [3].

Colchicin, das in der Gichttherapie eingesetzt wird, hemmt durch Blockade der Mikrotubuli die Mitose und die Migration von Phagozyten.

Unklar ist bisher, inwieweit die postulierte inhibitorische Wirkung von Colchicin auf die Kollagensynthese und die Kollagenaseaktivität als «antifibrosierende Wirkung» bedeutsam ist [4]. Weitere Erkenntnisse zur topischen Wirkung auf die Haut liegen ebenfalls nicht vor.

\section{Digitalis purpurea (roter Fingerhut)}

Diese aus der Familie der Scrophulariaceae stammende Pflanze ist in West- und Mitteleuropa weit verbreitet. Sie enthält eine Vielzahl von Glukosiden, Steroidsaponinen, Flavonoiden und Anthrachinonderivaten [5]. Die Digitalisglykoside werden inzwischen auch synthetisch hergestellt und sehr häufig in der Behandlung der Herzinsuffizienz eingesetzt.

Während die therapeutische Effektivität bei systemischer Gabe äusserst hoch und die therapeutische Breite recht schmal ist, ist die Wirkung bei topischer Anwendung weniger ausgeprägt. Hier soll es zu einer gewissen Tonisierung von oberflächlichen Venen kommen, so dass Digitalisextrakte traditionell bei venöser Insuffizienz, Thrombophlebitiden und postthrombotischen Syndromen eingesetzt wurden. Über die Resorptionsrate bei topischer Anwendung liegen keine hinreichenden Daten vor. Weiss [6] hebt die Wirkung von topischen Digitalispräparaten auf die Wundheilung hervor, ohne dass hier offenbar kontrollierte Studien vorgenommen wurden.

\section{KARGER}

Fax +497614520714

E-mail kargergmbh@aol.com

www.karger.com (c) 1999 S. Karger GmbH, Freiburg

Accessible online at:

http://BioMedNet.com/karger
Dr. Matthias Augustin

Universitäts-Hautklinik

Hauptstrasse 7

D-79104 Freiburg

Tel. +49761 270-6749, Fax 6829

E-mail augustin@haut.ukl.uni-freiburg.de 
Podophyllum peltatum (Fussblatt, Maiapfel)

Diese ursprünglich aus Nordamerika stammende Pflanze aus der Familie der Berberidaceae ist auch in der dermatologischen Therapie bekannt. Ihr Hauptwirkstoff Podophyllin weist eine antimykotische Wirkung aufgrund einer Interaktion mit dem Tubulin und nachfolgender Inhibition der Bildung von Mikrotubuli auf [7, 8]. Früher auch als Laxans verwendet, wird Podophyllin heute nur als 10-20\%ige Lösung zur Therapie von Condylomata acuminata eingesetzt.

\section{Hyoscyamus niger (Bilsenkraut)}

Diese Pflanze aus der Familie der Solanaceae ist in ganz Europa und den gemässigten Zonen der anderen Kontinente verbreitet.

Das Bilsenkraut enthält eine Vielzahl von pharmakologisch wirksamen Substanzen, besonders aber Alkaloide mit parasympatholytischer Wirkung (u.a. Hyoscyamin, Racemat des Atropins, Scopolamin) [9]. Diese Eigenschaften waren - ähnlich wie bei Belladonna - schon vor Jahrhunderten bekannt. Neben der parasympatholytischen Wirkung kommt es in bestimmten Konzentrationen und Zubereitungen auch zu halluzinogenen Effekten, so dass das Bilsenkraut auch als Rauschmittel verwendet wurde.

Der systemische Gebrauch des Bilsenkrautes ist heute obsolet. Reinformen des Scopolamins werden jedoch noch u. a. in der Ophthalmologie eingesetzt.

Die topische Anwendung von Scopolamin beschränkt sich ebenfalls auf die Augenheilkunde. Für die Anwendung bei Hauterkrankungen liegen keine zuverlässigen Angaben vor. Als Bestandteil von Narbencremes ist Bilsenkrautöl beschrieben [10], ohne dass hierfür eine wissenschaftliche Grundlage angegeben wird.

\section{Calendula officinalis (Ringelblume)}

Die aus der Familie der Asteraceae stammende und in weiten Teilen Europas verbreitete Pflanze wird in dermatologischen Präparaten sowie in kosmetischen Produkten häufig verwendet. Sie enthält multiple ätherische Öle, Flavonoide, Triterpensaponine, Carotinoide und Cumarine [11]. In vitro sowie in Tierversuchen sind antiphlogistische und granulationsfördernde Effekte beschrieben, ferner leichte antimikrobielle Wirkungen [12].

Demgemäss werden Ringelblumen-Zubereitungen in Salben zur Behandlung von Wunden und Ekzemen und zur Hautpflege eingesetzt.

\section{Eigenschaften von Unguentum lymphaticum}

In klinischen Berichten wird Unguentum lymphaticum eine schmerzlindernde, entstauende und entzündungshemmende Wirkung bei primären und sekundären Lymphödemen sowie bei Schwellungszuständen anderer Genese nachgesagt [13]. Berichtet wird auch über eine inhibitorische Wirkung auf die Bildung von Narben und Fibrosen [14].

\section{Experimentelle Studien zur Wirkung}

Zu Beginn der 80er Jahre beschrieben Casley-Smith et al. aufgrund von In-vitro-Versuchen eine stimulierende Wirkung auf Makrophagen [15]. Gefunden wurde in anderen Studien auch eine Aktivierung von dermalen Immunzellen (insbesondere Langerhans-Zellen) und Keratinozyten [16, 17].

Für Unguentum lymphaticum wird auch berichtet, dass es bei einer dreiwöchigen Behandlung von durch Lymphödemen indurierter Haut zu einer klinisch nachweislich weicheren Haut gekommen sei. Immunhistologische Untersuchungen der Haut zeigten einen leichten Anstieg der Dichte von CD68/HLA-DR-positiven Zellen in der papillären Dermis an [18]. Als Ursache wird eine unspezifische Stimulation dermaler Makrophagen angenommen [19].

\section{Klinische Studien zur Wirksamkeit}

In einer sehr umfangreichen klinischen Studie an über 7000 Patienten mit Lymphödemen fanden Földi et al. [20] günstige Wirkungen von Unguentum lymphaticum hinsichtlich Schmerzlinderung und Entstauung. Weitere Studien deuten auf eine Erhöhung des Lymphabflusses bei verschiedenen Ödemen hin [16, 17]. Postuliert wurde nach klinischen Versuchen auch eine deutliche antifibrotische Wirkung [14].

\section{Unerwünschte Wirkungen}

Die genaue Zahl der bisherigen Anwendungen von Unguentum lymphaticum ist nicht bekannt. Vermutlich sind seit der Markteinführung 1969 mehrere 10000 Patienten behandelt worden, ohne dass ernsthafte Nebenwirkungen oder Komplikationen berichtet wurden. Földi et al. berichten in einem klinischen Erfahrungsbericht [20], dass keine ernstzunehmenden oder unerwünschten Ereignisse und auch keine unerwünschten Interaktionen mit anderen Medikamenten oder Behandlungsansätzen auftraten. Kontaktallergische Reaktionen waren bei ca. 1,5 bis $2 \%$ der Patienten zu verzeichnen, wobei keine Angaben dazu vorliegen, welche Inhaltsstoffe zur Kontaktallergie geführt haben.

In toxikologischen Untersuchungen von Casley-Smith [21] konnten im Tierexperiment nach kutaner Applikation keine systemisch-toxischen Nebenwirkungen in akuten, subakuten und chronischen Beobachtungszeiträumen ermittelt werden. Keiner der kutan applizierten Wirkstoffe erreichte eine höhere Plasmakonzentration als $10 \mathrm{ppm}$. Im Verhältnis zu systemischen vasoaktiven Substanzen liegt hier somit eine günstige Relation zwischen Wirksamkeit an der Haut und toxisch bedeutsamen Wirkspiegeln vor.

Forsch Komplementärmed 1999;6(suppl 2):34-36 


\section{Bisherige Anwendungsgebiete und mögliche weite- re Indikationen}

Unguentum lymphaticum wurde bislang vornehmlich zur Behandlung des Lymphödems und anderer ödematöser Schwellungszustände eingesetzt. Bei diesen Indikationen liegt möglicherweise eine günstige Nutzen-Risiko-Relation vor, die einer Überprüfung in kontrollierten randomisierten Studien bedarf.

Aufgrund der postulierten antiödematösen Wirkungen kommt Unguentum lymphaticum potentiell auch für die Behandlung von postoperativen und posttraumatischen Ödemen sowie zur topischen Behandlung von Schwellungen bei chronisch-venöser Insuffizienz in Frage. Eine weitere interessante Indikation, die an Berichte über eine antifibrotische Wirkung anknüpft, ist die Behandlung von Dermatosklerosen und Narben. Als Erkrankungen kämen hier z. B. die Dermatoliposklerose und die Atrophie blanche bei der CVI sowie die Morphea, die systemische Sklerodermie und der Lichen sclerosus et atrophicus in Frage.

Die ermittelte aktivierende Wirkung auf Makrophagen und Keratinozyten lässt an einen Einsatz bei erregerbedingten Hauterkrankungen sowie in der Wundheilung denken, ohne dass hier bislang ausreichende klinische Voruntersuchungen vorgenommen wurden.
Insgesamt bedürfen alle genannten Indikationen einer eingehenden Kontrolle durch wissenschaftliche Studien.

\section{Fazit und Ausblick}

Mit der Salbe Unguentum lymphaticum liegt eine Präparation aus pharmakologisch wirksamen Pflanzenextrakten vor, die sich bei offenen klinischen Beobachtungen in der Lymphödem-Therapie als geeignet erwiesen hat. Erste experimentelle Untersuchungen machen die Salbe in der vorliegenden Präparation zu einem Kandidaten für weitergehende Testungen bei der Indikation «Lymphödem» wie auch bei anderen Erkrankungszuständen der Haut.

In Ergänzung zu den klinischen Untersuchungen am Gesamtpräparat sind aus wissenschaftlicher Sicht auch die Wirkungen der Einzelkomponenten von Interesse.

Die Literatur kann beim Verfasser angefordert werden. 